\title{
Ionospheric scintillation modeling for high- and mid-latitude using B-spline technique
}

\author{
S. Priyadarshi ${ }^{1}$
}

Received: 12 March 2015 / Accepted: 22 July 2015 / Published online: 5 August 2015

(C) The Author(s) 2015. This article is published with open access at Springerlink.com

\begin{abstract}
Ionospheric scintillation is a significant component of space-weather studies and serves as an estimate for the level of perturbation in the satellite radio wave signal caused due to small-scale ionospheric irregularities. B-spline functions are used on the GPS ground based data collected during the year 2007-2012 for modeling high- and mid-latitude ionospheric scintillation. Proposed model is for Hornsund, Svalbard and Warsaw, Poland. The input data used in this model were recorded by GSV 4004b receivers. For validation, results of this model are compared with the observation and other existing models. Physical behavior of the ionospheric scintillation during different seasons and geomagnetic conditions are discussed well. Model is found in good coherence with the ionospheric scintillation theory as well as to the accepted scintillation mechanism for high- and mid-latitude.
\end{abstract}

Keywords Ionosphere - Scintillation · B-spline · GPS · Small scale irregularities

\section{Introduction}

Ionospheric scintillation is a phenomenon of special interest to space scientists and navigational satellite systems users. It has been widely discussed and studied in the past but, still difficult to model and predict on large scales. Ionospheric scintillations are caused due to the random electron density irregularities acting as wave scatterers (Wernik et al. 2004). As the signal propagation continues after passing through

\footnotetext{
S. Priyadarshi

spriyadarshi@cbk.waw.pl

1 Space Research Centre (SRC), Polish Academy of Science (PAS), Bartycka 18a, 00-716 Warsaw, Poland
}

the region of irregularities in the ionosphere, phase and amplitude scintillation develops through interference of scattered rays (Kintner et al. 2007).

Climatological models are constructed to study dependencies of the strength and/or occurrence of scintillation on local time, season, solar and magnetic activity. TRANSMIT NEWSLETTER Issue 1 (2012) summarizes very briefly the important models of ionospheric scintillation that have been developed for improving GNSS vulnerability to space weather. The first empirical model of scintillation was proposed by Fremouw and Rino in 1973. This model could estimate the scintillation index $S_{4}$ being the measure of the scintillation intensity on VHF/UHF, under weak scatter conditions roughly identified with weak scintillation. A weak scatter condition is often exceeded near the equatorial anomaly and auroral regions. This model led to the foundation of a more advanced model "WBMOD".

Aarons developed an analytic model in 1985 using 15-min peak to peak scintillation indices (not $S_{4}$ ) taken over 5 years at Huancayo, Peru using LES 6 satellite transmitted at $254 \mathrm{MHz}$ (Aarons 1985). Later came the India model by Iyer and his group in 2006 (Iyer et al. 2006). They used a cubic-B spline technique to develop an empirical model of magnetic quiet time scintillation occurrence at Indian equatorial and low latitudes. A $250 \mathrm{MHz}$ signal from FLEETSAT satellite was measured for 2 years at Trivandrum, near the magnetic equator, and at Rajkot at the crest of equatorial anomaly. To describe the structure and extent of the radio scintillation generated by turbulence around and within the equatorial plumes a physical model has been developed by J.M. Retterer (2010).

The first climatological model WBMOD was developed by Northwest Research Associates, Inc. in which the user can specify his operating scenario. As the output the model returns: the phase scintillation spectral index $p$, the spec- 
tral strength parameter $T, S_{4}$, and rms phase $\sigma_{\varphi}$. Another, Global Ionospheric Scintillation Model (GISM), has been described by Beniguel and Buonomo in 1999. The model consists of two parts; the NeQuick model and the scintillation model based on a multiple phase screen algorithm and a second part which needs statistical information about irregularities as input. The algorithm is used to calculate the scintillation index at the receiver.

Basu and her group used in situ satellite data in scintillation modeling for the first time in 1976. They assumed a $3 \mathrm{D}$ power law irregularity spectrum with a constant spectral index of 4. They prepared another high latitude scintillation model (1988) using Atmospheric Explorer D data. Due to a limited availability of data the model was only suitable for northern winter under sunspot minimum condition. Wernik et al. (2007) used the Dynamics Explorer B data to estimate the irregularity spectral index and turbulence strength parameter, the factors that are required to calculate the scintillation index (Rino 1979). Their approach has been extended by Liu et al. (2012) by introducing the finite outer scale.

Models listed above reproduce well enough the global morphology of ionospheric scintillations but they often show lack of precession in detailed resolution for short time period (e.g. geophysical case studies). We will dislodge this modeling limitation by using B-spline function as a modeling tool. B-spline functions are well known for preserving the local trends of the data set even if the data is compressed on time and spatial scale. In this paper we are presenting an empirical climatological model. This model is based on the ground based GSV4004b receiver data recorded at Hornsund, Svalbard and Warsaw, Poland since 2007. We will discuss here the preparation of the data set used in this model. Great emphasis has been given to the B-spline technique used for building up the present model. For validating our model we have presented few figures equating results obtained from the model and from the observation. Results and Discussion section present and discuss seasonal and geophysical behavior of scintillation index. Conclusion section discusses merits and limitations of our model. This part is also dedicated to convince the readers of the usefulness of B-spline functions for modeling purposes.

\section{Derivation of the B-spline ionospheric scintillation model}

This model have been prepared in the scope of studying changes in scintillation behavior when one moves from midlatitude to high-latitude. The model has been tested for amplitude scintillation for mid- and high-latitude stations Warsaw, Poland and Hornsund, Svalbard respectively. For midlatitude where scintillation occurrence is quiet rare, amplitude scintillation index is the best indicator as compared to phase scintillation. Previous workers (e.g. Wernik et al. 2004; Basu and Basu 1993 and references therein) have pointed out that if the electron density fluctuations are significant, then the phase fluctuations can be so large that the wave is no longer coherent and the interference of rays is not possible. Analysis shows that when the phase fluctuations reach a certain limiting value, the intensity of amplitude scintillation ceases to increase. Taking all these points into consideration we decided to move forward with the amplitude scintillation modeling only. We built a model for mid-latitude and high-latitude stations using ground based GSV 4004b receiver data situated at Warsaw, Poland and Hornsund, Svalbard respectively. We are using B-spline basis function of order 4 for ground based measurements. Ionospheric scintillation index as a function of local time, day/season/month, geographic coordinates, $K_{p}$ index and solar flux value $F 10.7$, is expressed as product of univariate normalized B-splines as given below

$S_{4}\left(t, d\right.$, geo.coord. $\left.k_{p}, F 10.7\right)$

$$
\begin{aligned}
& =\sum_{i=1}^{24} \sum_{j=1}^{365} \sum_{k=1}^{9} \sum_{l=1}^{6} a_{i, j, k, l} \\
& \quad * N_{i, 4}(t) N_{j, 4}(d) N_{k, 4}\left(k_{p}\right) N_{l, 4}(F 10.7)
\end{aligned}
$$

In Eq. (1), $a_{i, j, k, l}$ are monthly mean of amplitude scintillation index recorded by GPS GSV 4004b receiver for each interval of magnetic local time, invariant coordinate, $K_{p}$ index and solar radio flux $F 10.7 \mathrm{~cm} . N_{i, 4}, N_{j, 4}, N_{k, 4}$ and $N_{l, 4}$ are b-spline basis function of degree 4 . We are using NASA OMINIWEB data server (http://omniweb.gsfc.nasa.gov/) for getting geophysical index (e.g. $K_{p}, F 10.7$ etc.). For comparing modeled $S_{4}$ with the $S_{4}$ calculated using observations, GPS data must be reduced to the overhead value by correcting for the propagation geometry as discussed by Priyadarshi and Wernik (2013). $S_{4}$ index is computed as the standard deviation of the received signal power normalized to the average signal power. We have followed Van Dierendonck et al. (1993), for removing the effects of ambient noise from $S_{4}$. The corrected $S_{4}$ is given by Eq. (2) below

$S_{4}($ corrected $)=\frac{\left(S_{4 T}^{2}-S_{4 N}^{2}\right)^{1 / 2}}{\left[\sin \left(E^{\prime}\right)\right]^{-\mu}}$,

where $S_{4 T}$ is the original, measured scintillation index and $S_{4 N}$ is the ambient noise scintillation index. As suggested by Priyadarshi and Wernik (2013), we have used the correction indices $\mu=2.59$ and 2.95, for Warsaw and Hornsund, respectively. $E^{\prime}$ is the elevation angle at the pierce point and is related to the local elevation angle $E$ at the receiver (ElArini et al. 1994)

$$
E^{\prime}=\sin ^{-1}\left(\left[1-\left(R_{e} \cos (E) /\left(R_{e}+h\right)\right)^{2}\right]^{-1 / 2}\right) .
$$


We calculated zenith angle using $E^{\prime}$ and azimuth angle $\left(A_{z}\right)$ using formula (3)

Zenith angle $(\theta)=E^{\prime}-\sin ^{-1}\left[\frac{\cos \left(E^{\prime}\right) * R_{e}}{\left(R_{e}+h\right)}\right]$.

Geographic latitude and longitude at the pierce point are calculated (Knight 2000)

Latitude $\left(\alpha^{\prime}\right)=\alpha+\theta \cos (A z)$

Longitude $\left(\beta^{\prime}\right)=\beta+\theta \frac{\sin (A z)}{\cos \left(\alpha^{\prime}\right)}$

where $\alpha$ and $\beta$ are geographic latitude and geographic longitude of the ground based observing station. We used a MATLAB subroutine which uses IGRF model for calculating corrected geomagnetic coordinates and MLT for both of the data stations.

The outcomes of the present model have been compared with other relevant models, case studies and observations. In Sect. 3 model outcomes have been discussed for Hornsund Svalbard and its similarity with the outcomes of Gola et al. (1992). In Sect. 4 our results and possible physical mechanism have been discussed along with the outcomes of many pre-existing scintillation models and case studies (e.g. Wernik et al. 2004, 2007; Coster et al. 2005; Gola et al. 1992 etc.). Such parallel discussion validates our model. Direct numerical comparison (which is mostly done in review articles) is beyond the scope of this paper since we are proposing here our model and trying to preserve the local trends of the input data using B-spline technique.

\section{Results and discussion}

\subsection{Intensity scintillation model for Hornsund}

We have used the corrected amplitude scintillation index for this modeling. The range of calculated $S_{4}$ used in the present model was between 0 to 1.5 . We considered the data only for elevation angle $>30$ degree. Figure 1 shows the histogram of the data value for different seasons used in this study. From the histogram it seems that number of data falling in this shorting range are maximum in equinox and approximately equal in summer and winter months.

Equinox results The location of Polish Polar Station is at Hornsund, Spitsbergen (77 deg. N, 15.55 deg. E). The invariant latitude $\Lambda=73.4 \mathrm{deg}$. makes us capable to observe satellite transmission penetrating the ionosphere depending on geophysical conditions in the auroral zone as well as polar cap region. As we see in Fig. 2 our data cover 70 deg. to $80 \mathrm{deg}$. corrected geomagnetic latitude (CGML).
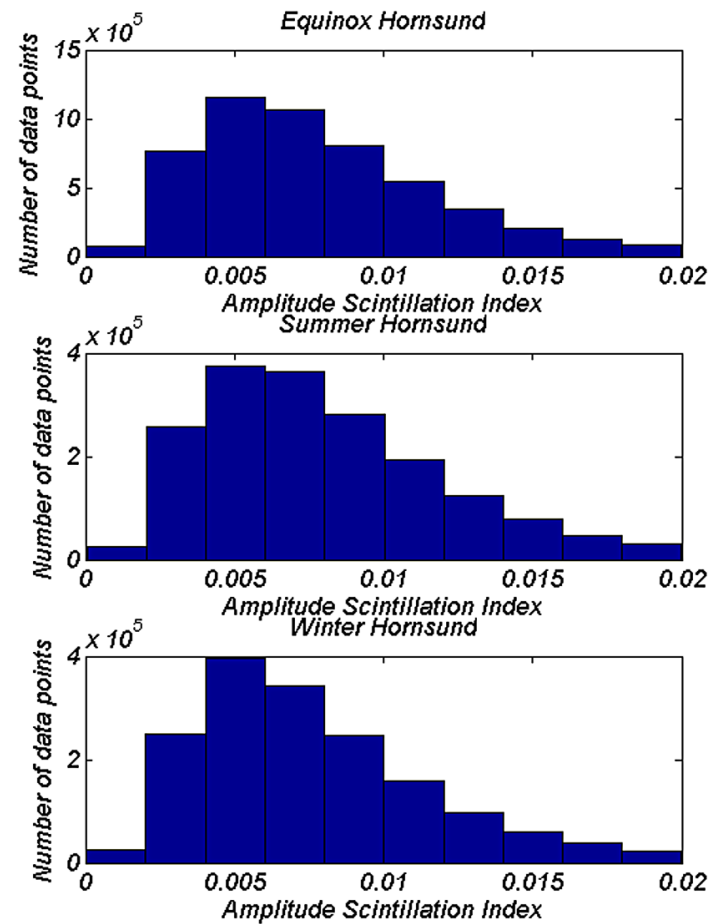

Fig. 1 Histogram showing data density of Hornsund Svalbard data for equinox, summer and winter

Ionospheric scintillation index increases with increasing geomagnetic latitude. We observe reduction in scintillation index near the location of the ground based station. Gola et al. (1992) observed a maxima near invariant latitude of the Polish Polar Station. The maxima in their case was due to geometrical effect of wave propagation through irregularity in magnetic zenith which was taken care of but not as accurately as in the present paper. We have corrected our data considering $S_{4}$ index as a power-law function of the form irregularity and using the appropriate correction index for geometry of propagation accordingly.

The minimum in $S_{4}$ is more visible during geomagnetic disturbed conditions in both models as well as observations. In fact either minimum or maximum at the station latitude might be indicative of not full correction for geometry. Assuming that the correction is right minimum could be an ionospheric trough between the auroral oval and polar cap regions. Another most important conclusion one can extract from Fig. 2 is, the map created through MATLAB linear interpolation method of calculated $S_{4}$, are in good agreement with the maps which are prepared using reproduced scintillation index $S_{4}$, from the B-spline model.

Summer results Figure 3 shows the corrected scintillation behaviour during summer. The level of scintillation is a little higher than that in equinox and henceforth there is an enhancement in scintillation index near the magnetic noon as well. Minimum in scintillation index is observed near 

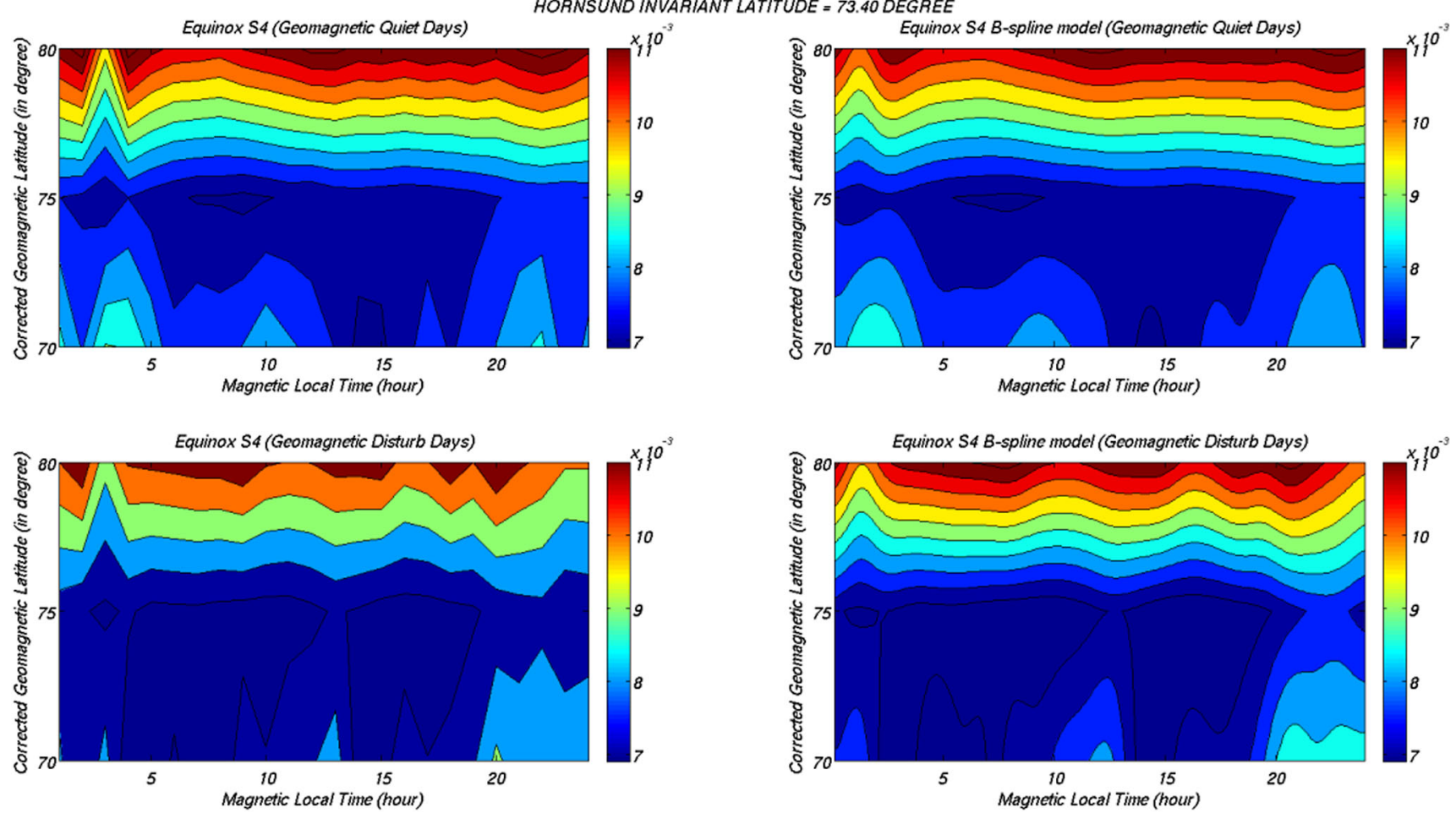

Fig. 2 Corrected $S_{4}$ index for equinox months for $K_{p} \leq 3$ (Geomagnetic Quiet Days) and $K_{p}>3$ (Geomagnetic Disturbed Days). Left contour is prepared through linear interpolation of $S_{4}$ calculated from the observations and right maps are prepared from the reproduced results of the model
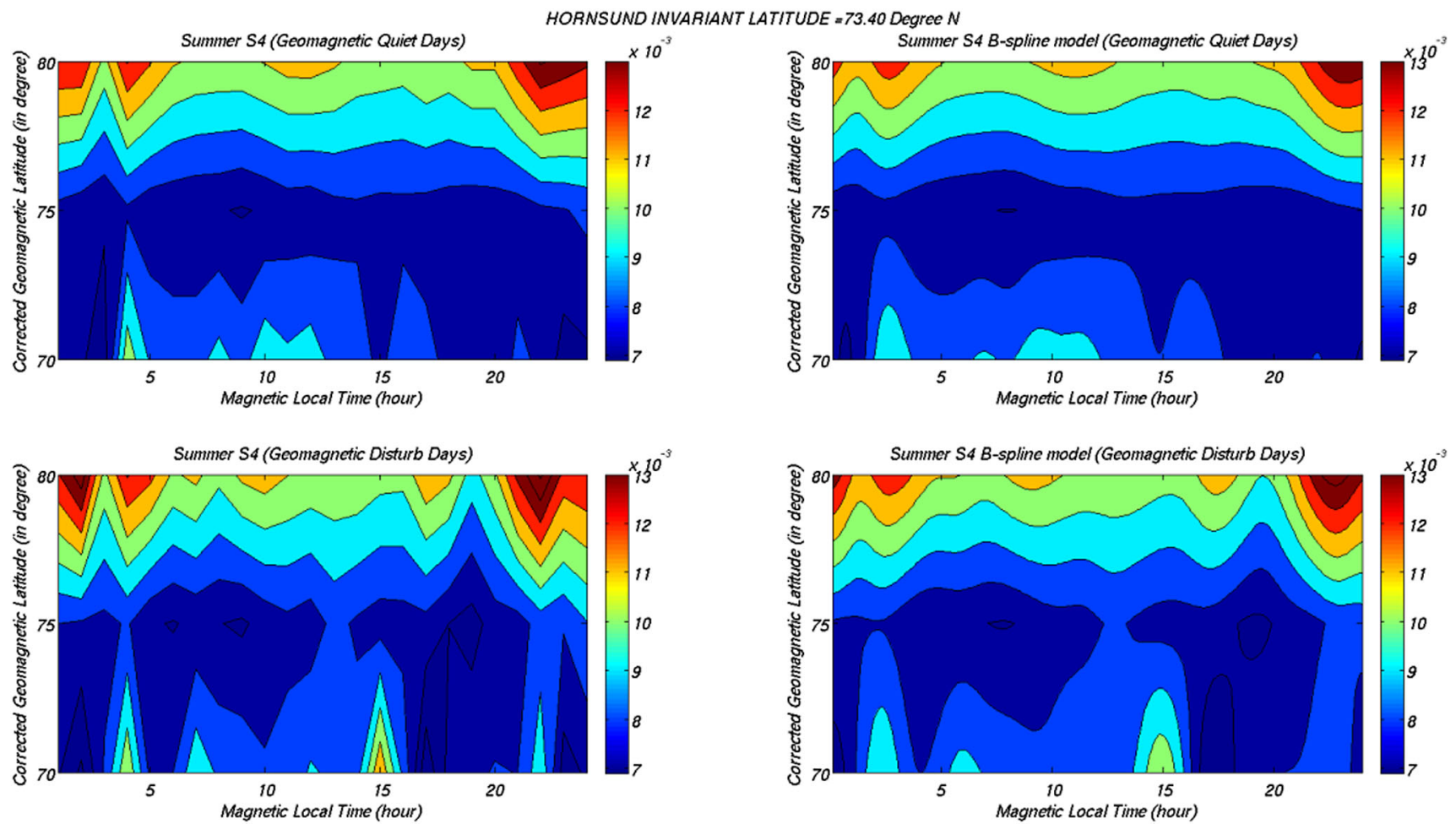

Fig. 3 Corrected $S_{4}$ index for summer months for $K_{p} \leq 3$ (Geomagnetic Quiet Days) and $K_{p}>3$ (Geomagnetic Disturbed Days). Left contour is prepared through linear interpolation of $S_{4}$ calculated from the observations and right maps are prepared from the reproduced results of the model 

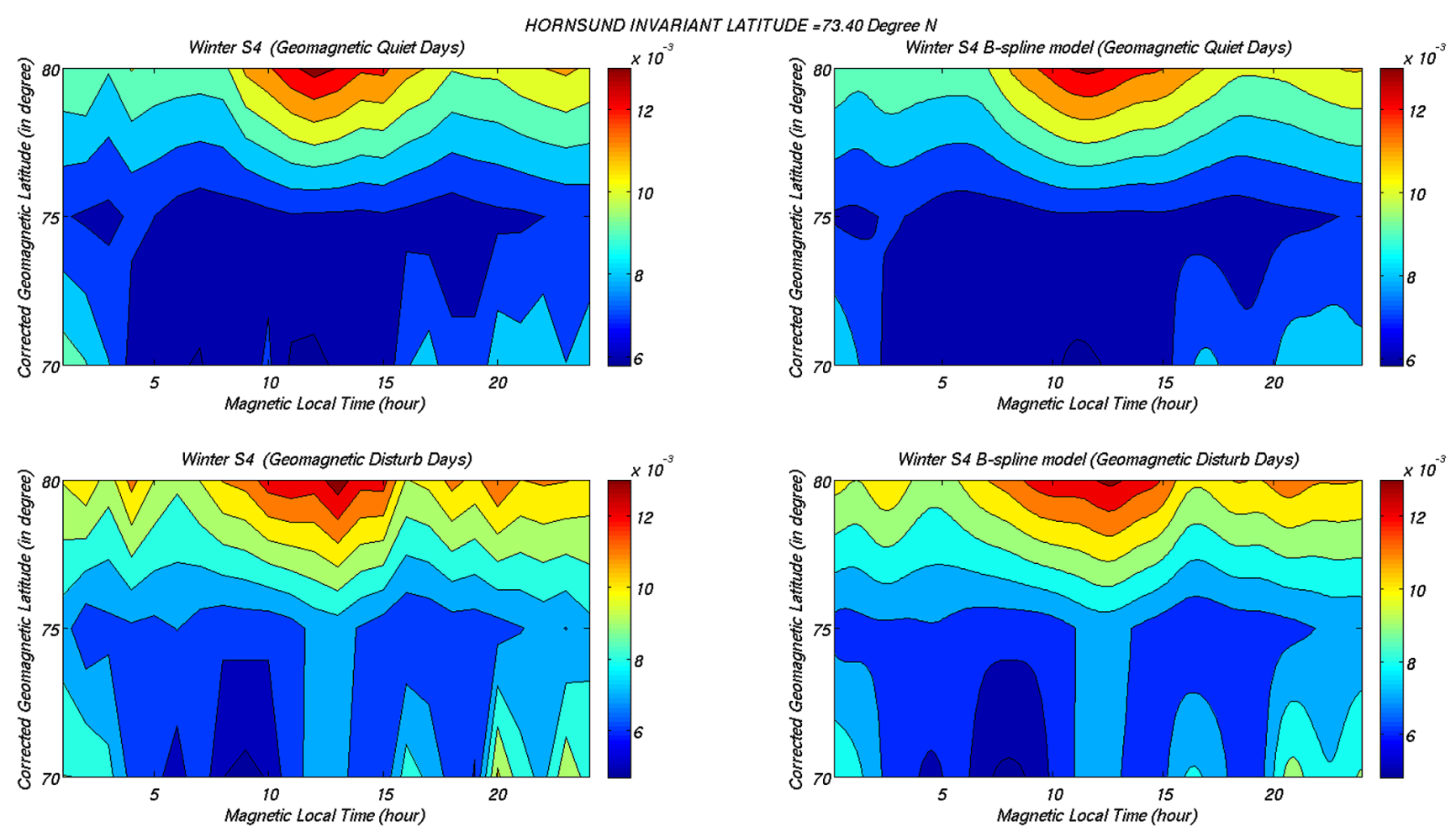

Fig. 4 Corrected $S_{4}$ index for winter months for $K_{p} \leq 3$ (Geomagnetic Quiet Days) and $K_{p}>3$ (Geomagnetic Disturbed Days). Left contour is prepared through linear interpolation of $S_{4}$ calculated from the observations and right maps are prepared from the reproduced results of the model

73 deg. invariant latitude. This minimum is continuous with local time for geomagnetic quiet days but during geomagnetic disturbed days a little discontinuity is observed near magnetic noon. The common feature between summer and equinox maps is the enhancement in scintillation index with CGML latitude.

From both figures it is clear that during summer as well as in equinox outside the auroral oval ( $\approx 70 \mathrm{deg}$. CGML) scintillation level depends weakly on MLT. This result is fully consistent with Gola et al. (1992). During summer months scintillation pattern gets a bit complicated for magnetic active periods. Most intense scintillation pattern expands during magnetic midnight and magnetic noon hours.

Winter results The scintillation level is slightly higher than that in equinox. Figure 4 shows the maps for winter corrected $S_{4}$ prepared using MATLAB linear interpolation as well as $S_{4}$ index given by the model. The winter results are more interesting during geomagnetic disturbed days. A reasonable scintillation enhancement is visible equatorward side during magnetic noon and midnight. The region of strongest scintillation is located at polward boundary.

During geomagnetic quiet condition enhancement in scintillation index at magnetic moon is more intense while, during geomagnetic disturbed days, scintillation index is strongly dependent on MLT. The geomagnetic disturbed pat- tern spreads latitudinally at magnetic noon hours and dusk regions. This spread disappears during and after midnight.

Near 73 deg. invariant latitude and geomagnetic disturbed period scintillation depends on MLT but, scintillation variation seems independent of MLT for geomagnetic quite condition. At high magnetic activity polar cap boundary of scintillation coincides with auroral boundary (Gola et al. 1992). This is due to the expansion of scintillation pattern as a function of MLT.

\subsection{Intensity scintillation model for Warsaw}

Receiver is located at Warsaw, Poland (52.23 deg. N, 21.01 E deg., invariant latitude $\Lambda=46.70 \mathrm{deg}$.). GPS scintillation at mid-latitude are often associated with magnetic storms near solar maximum. Fortunately, the data set we are using is for near solar maximum period (from the year 2011 to 2012). Here we will study and discuss seasonal and geophysical morphology of scintillation. Our data set covers $45 \mathrm{deg}$. to $55 \mathrm{deg}$. of CGML latitude. Corrected $S_{4}$ we have chosen for this study range between 0 to 1.5 . To remove the effect of multipath we have taken into account elevation angle only greater than 30 degree.

Equinox results Figure 5 shows the equinox behaviour of scintillation during geomagnetic quiet and geomagnetic disturbed conditions. For quiet geomagnetic condition scintil- 

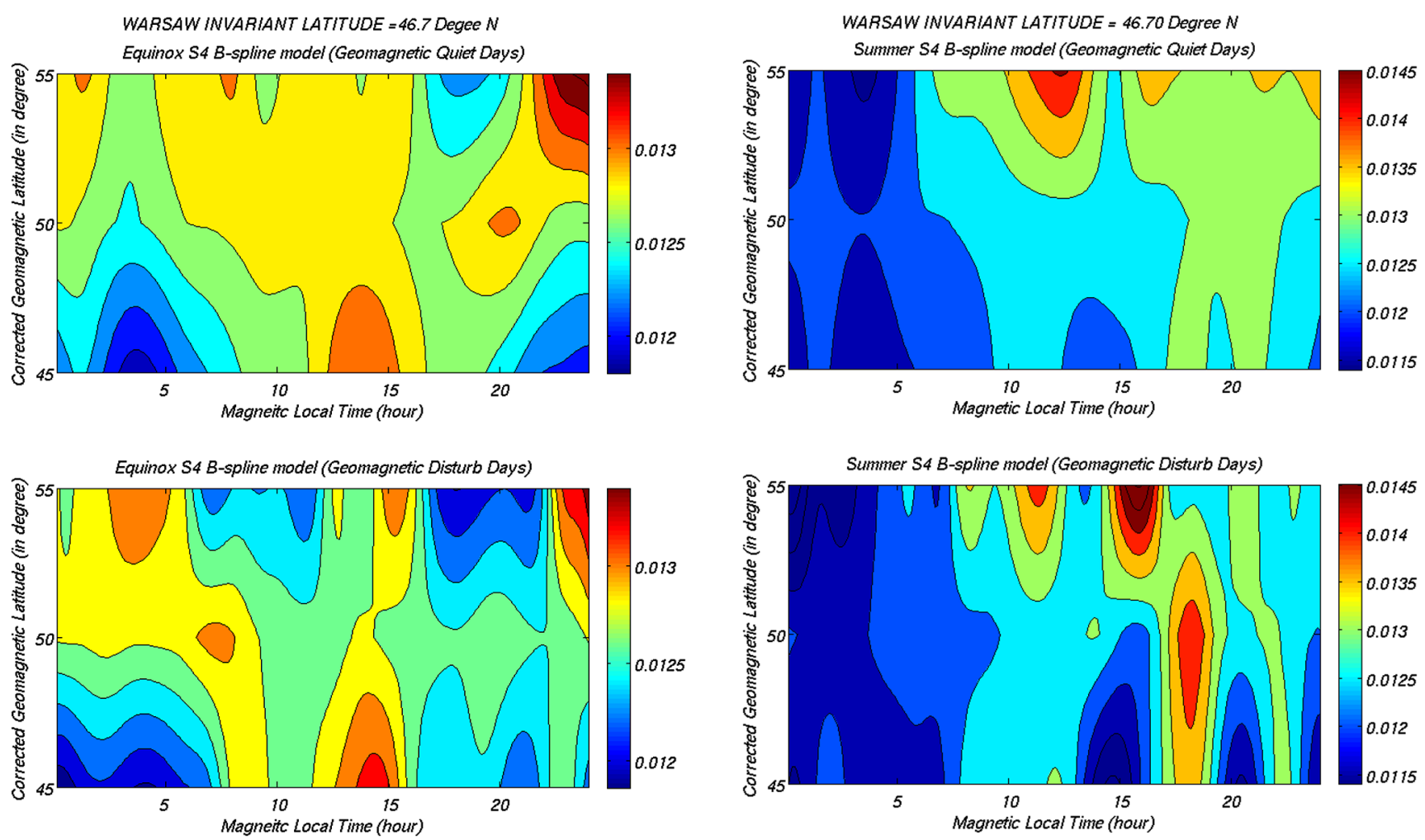

Fig. 5 Corrected $S_{4}$ index for equinox months $K_{p} \leq 3$ (Geomagnetic Quiet Days) and $K_{p}>3$ (Geomagnetic Disturbed Days)

lation index seems strongly dependent on magnetic time as it shows maxima near midnight hours as well as at magnetic noon but, for geomagnetic disturbed condition $S_{4}$ is weakly dependent on magnetic time.

Scintillation spread during geomagnetic quiet condition is more visible near magnetic noon and dusk sectors. At magnetic noon the scintillation spread is equarward. Scintillation phenomenon is more defined at midnight time for CGML latitude $\geq 50$ degree. For strong geomagnetic condition spread is more defined near midnight regions than near magnetic noon times. Near dusk sector a maxima like stretched mango is visible during the magnetic quite conditions which completely disappears for the magnetic disturbed conditions. The level of scintillation index is lowest during the equinox months. Near invariant latitude $(\approx 47$ deg.) of observing station scintillation enhancement weakly follows MLT.

Summer modeling results for Warsaw During summer we observed the strongest scintillation level. The maximum is visible only near magnetic noon for CGML latitude $\geq 50 \mathrm{deg}$. With increasing geomagnetic activity the regions of the most intense scintillation expand from magnetic noon to magnetic dusk sector. Figure 6 shows the contour map for summer for geomagnetic quiet and disturbed conditions. A faint maxima during magnetic noon is observed at about

Fig. 6 Corrected $S_{4}$ index for summer months $K_{p} \leq 3$ (Geomagnetic Quiet Days) and $K_{p}>3$ (Geomagnetic Disturbed Days)

$47 \mathrm{deg}$. of CGML latitude which intensifies for strong geomagnetic condition. With increasing geomagnetic activity the maxima spread from magnetic noon to dusk sector.

Winter modeling results for Warsaw Figure 7 shows the corrected $S_{4}$ maps for Warsaw during winter months. The upper map shows geomagnetic quiet condition while lower is for geomagnetic disturbed condition. The winter results are interesting since they show full variation with MLT. Scintillation get intensified before the magnetic noon hours. Scintillation spread is equatorward and more defined before the magnetic noon hours.

With increasing magnetic activity we observe an intensification in scintillation index for CGML latitude $>50$ degree which is visible in dusk sector. Dawn sectors also shows intense maxima with the increase in geomagnetic activity.

\section{Summary and conclusion}

The model prepared for the Warsaw and Hornsund uses B-spline basis function of degree 4 . The reason for using only 4th degree basis function is to increase the number of data points within each bin of averaged data set. B-spline is an effective technique because of full user control and there is always possibility of improvement in the model. Up to so 

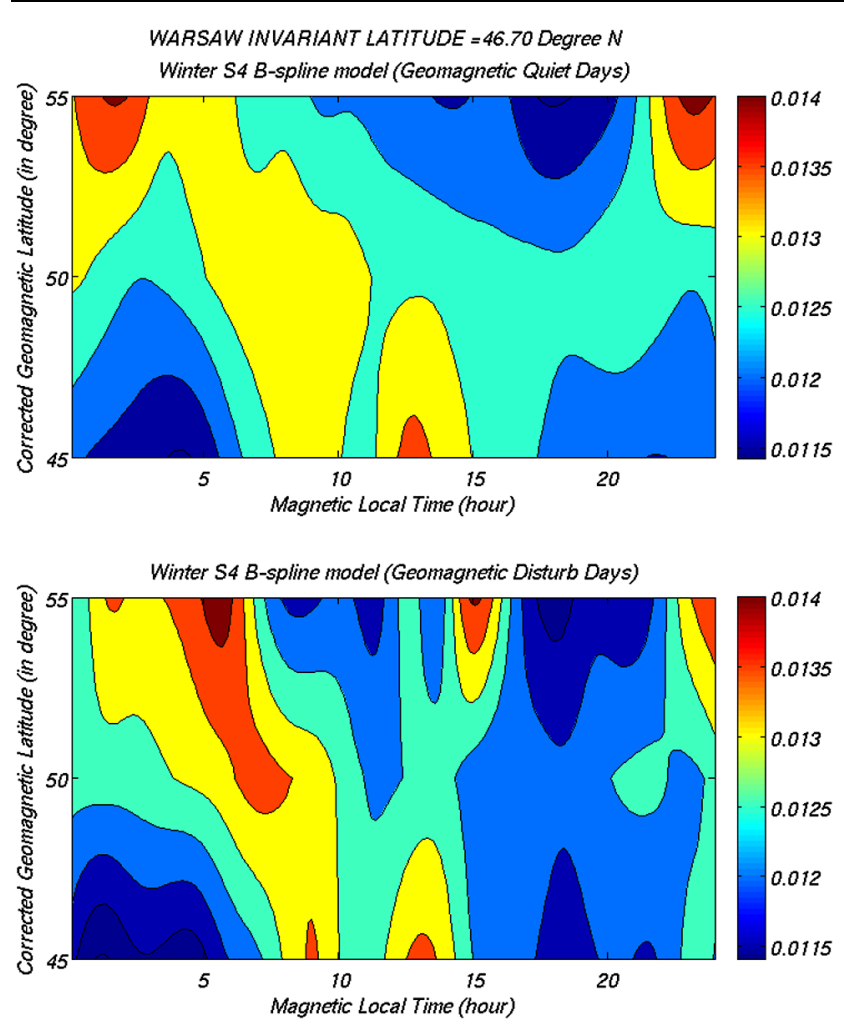

Fig. 7 Corrected $S_{4}$ index for winter months $K_{p} \leq 3$ (Geomagnetic Quiet Days) and $K_{p}>3$ (Geomagnetic Disturbed Days)

far we have built a model in corrected geomagnetic coordinates only. Our results are in good coherence with the observation. Our results provide a clear indication of changes in scintillation morphology when we move from the mid latitude region to the high latitude region. Therefore we say here strictly that our model is validated. For Hornsund scintillation index, for the CGM latitude $\left(\Lambda^{\circ}\right)>75^{\circ}$ is varying independently with respect to the MLT and geomagnetic activity. This may be due to large scale irregularity. The stagnant variation in scintillation pattern during equinox seems due to long life time of large scale (hundreds of meters to kilometers) irregularity and their convection from the places of origin (Aarons 1982). Ionospheric irregularities present in the high latitude ionosphere are kept in motion by phenomena that couple momentum and energy from the solar wind into the Earth's magnetosphere. The convection of irregularities is controlled by interplanetary magnetic field (IMF) (Kelley et al. 1982). Coupling occurs where the magnetic fields of the magnetosheath and magnetosphere are antiparallel. During southward orientation of the IMF (i.e. $B_{z-}$ ), coupling occurs on the subsolar magnetopause near the noon MLT meridian. For azimuthal IMF (i.e., $B_{y+}$ or $B_{y-}$ ), the location of coupling in the northern hemisphere shifts either towards dusk or dawn, respectively (Ruohoniemi and Greenwald 2005). While during summer most intense scintillation is observed near magnetic midnight and magnetic noon.
With increasing geomagnetic activity, scintillation pattern spreads near magnetic midnight and magnetic dusk regions as evident in Fig. 3. The possible explanation for this is, during the summer, high E-region conductivity slows the instability growth rate and increases the cross-field plasma diffusion, which removes small-scale structures already at a short distance from the source (corrected geomagnetic latitudes corresponding to the magnetic midnight and magnetic noon) region (Kivanç and Heelis 1998). But in the cusp and night time auroral regions medium and large scale structures are created by structured fluxes of precipitated electrons. With increasing magnetic activity and in some situation these medium and large scale structures may produce small scale structures through the $E \times B$ or current-convective instability (Wernik et al. 2004). For geomagnetic quiet condition during winters the scintillation index for the CGM latitude $\left(\Lambda^{\circ}\right)>75^{\circ}$ scintillation index maximizes near magnetic noon followed by magnetic dusk and magnetic night time regions. As the magnetic activity increases, scintillation index start following MLT. During winters the convecting structures decay less rapidly and give rise to scintillation patterns. But, with increased geomagnetic activity it is possible that these decaying convecting structures may form small scale structures through $E \times B$ instability which follow MLT (Gola et al. 1992).

For Warsaw we observed that during equinox and weak magnetic condition scintillation pattern for CGML latitude $\geq 50$ degree are strongly dependent on MLT. But, with increasing magnetic activity scintillation pattern shows weak dependence on MLT. Main cause of scintillation at midlatitudes is inner-magnetospheric electric field creating irregularities on pre-existing ionospheric density gradients and local instabilities operating on pre-existing ionospheric density gradients (Coster et al. 2005). With increase in geomagnetic field, regions hosting large ionospheric density gradient last longer since, they are associated with high velocity ionospheric flows. In summer we observe strongest scintillation as compared to the other seasons. During the full sunlit days of summer months, E-region conductivity increases due to photo ionization. This may contribute to the enhanced inner-magnetospheric electric fields which reveal rapidly fluctuating plasma drifts (Wernik et al. 2007). Scintillation patterns follow MLT during winter months and shows equatorward spread with magnetic field enhancement. Taking $E \times B$ instability mechanism into account winter scintillation pattern could be explained quite satisfactorily. The $E \times B$ instability is the only cause responsible for producing Fresnel scale irregularities (Mishin et al. 2003a, 2003b). The Poynting vector represents the directional energy flux density of an electromagnetic field. This vector can be in both directions upwards and downward. Few times the electric field can be in coherence with the density fluctuations. 
Acknowledgements This research work is undertaken in the scope of the TRANSMIT ITN (www.transmit-ionosphere.net), funded by the Research Executive Agency within the 7th Framework Program of the European Commission, People Program, Initial Training Network, Marie Curie Actions-GA No. 264476. I am grateful to late Professor Andrzej W. Wernik of Space Research Poland, for his guidance, and support during the preparation of this paper.

Open Access This article is distributed under the terms of the Creative Commons Attribution 4.0 International License (http://creative commons.org/licenses/by/4.0/), which permits unrestricted use, distribution, and reproduction in any medium, provided you give appropriate credit to the original author(s) and the source, provide a link to the Creative Commons license, and indicate if changes were made.

\section{References}

Aarons, J.: Global morphology of ionospheric scintillations. Proc. IEEE 70(4), 360-378 (1982)

Aarons, J.: Construction of a model of equatorial scintillation intensity. Radio Sci. 20, 397-402 (1985)

Basu, S., Basu, S.: Ionospheric structures and scintillation spectra. In: Tatarski, V.I., Ishimaru, A., Zavorotny, V.U. (eds.) Wave Propagation in Random Media (Scin-tillation), pp. 139-153. The International Society for Optical Engineering, Bellingham (1993)

Basu, Su, Basu, Sa., Khan, B.K.: Model of equatorial scintillation from in-situ measurements. Radio Sci. 11, 821-832 (1976)

Basu, Su, Basu, Sa., Weber, E.J., Coley, W.R.: Case study of polar cap scintillation modeling using DE 2 irregularity measurements at 800 km. Radio Sci. 23, 545-553 (1988)

Coster, A., Skone, S., Mitchell, C., De Franceschi, G., Alfonso, L., Romano, V.: Global studies of GPS scintillation. Paper presented at National Technical Meeting (NTM 2005). Inst. of Navig., San Diego (2005)

El-Arini, M.B., Conker, R.S., Albertson, T.W., Reagan, J.k., Klobuchar, J.A., Doherty, P.H.: Comparison of real-time ionospheric algorithms for a GPS Wide-Area Augmentation System (WAAS). Navigation 41(4), 393-413 (1994)

Fremouw, E.J., Rino, C.L.: An empirical model for average F-layer scintillation at VHF/UGF. Radio Sci. 8, 213-222 (1973)

Gola, M., Wernik, A.W., Franke, S.J., Liu, C.H., Yeh, K.C.: Behaviour of HILAT scintillation over Spitsbergen. J. Atmos. Terr. Phys. 54(9), 1207-1213 (1992). doi:10.1016/0021-9169(92)90146-C

Iyer, K.N., Souza, J.R., Pathan, B.M., Abdu, M.A., Jivani, M.N., Joshi, H.P.: A model of equatorial and low latitude VHF scintillation in India. Indian J. Radio Space Phys. 35, 95-104 (2006)
Kelley, M.C., Vickrey, J.F., Carlson, C.W., Torbet, R.: On the origin and spatial extent of high-latitude $\mathrm{F}$ region irregularities. J. Geophys. Res. 87, 4469-4475 (1982)

Kintner, P.M., Ledvina, B.M., de Paula, E.R.: GPS and ionospheric scintillations. Space Weather 5, S09003 (2007). doi:10.1029/ 2006SW000260

Kivanç, Ö., Heelis, R.A.: Spatial distribution of ionospheric plasma and field structures in the high-latitude F region. J. Geophys. Res. 103, 6955-6968 (1998)

Knight, M.F.: Ionospheric Scintillation Effects on Global Positioning System Receivers. Ph.D. Thesis, Department of Electrical and Electronic Engineering, Faculty of Engineering, The University of Adelaide, Adelaide, South Australia (2000)

Liu, Y.H., Liu, C.H., Su, S.Y.: Global and seasonal scintillation morphology in the equatorial region derived from ROCSAT-1 in-situ data. Terr. Atmos. Ocean. Sci. 23(1), 95-106 (2012)

Mishin, E.V., Burke, W.J., Huang, C.Y., Rich, F.J.: Electromagnetic wave structures within subauroral polarization streams. J. Geophys. Res. 108 (2003a). doi:10.1029/2002JA009793

Mishin, E.V., Burke, W.J., Basu, S., Basu, S., Kintner, P.M., Ledvina, B.: Stormtime ionospheric irregularities in SPAS related troughs: Causes of GPS scintillations at mid-latitudes. Eos Trans. AGU 84(46). Fall Meet. Suppl., Abstract SH52A-07 (2003b)

Priyadarshi, S.: Review of existing scintillation models. TRANSMIT NEWSLETTER, issue 1 November 2012 (2012)

Priyadarshi, S., Wernik, A.W.: Variation of the ionospheric scintillation index with elevation angle of the transmitter. Acta Geophys. (2013). doi:10.2478/s11600-013-0123-3

Retterer, J.M.: Forecasting low-latitude radio scintillation with 3-D ionospheric plume models: 2. Scintillation calculation. J. Geophys. Res. 115, A03307 (2010). doi:10.1029/ 2008JA013840

Rino, C.L.: A power law phase screen model for ionospheric scintillation, 1. Weak scatter. Radio Sci. 14, 1135-1145 (1979)

Ruohoniemi, J.M., Greenwald, R.A.: Dependencies of high-latitude plasma convection: Consideration of interplanetary magnetic field, seasonal, and universal time factors in statistical patterns. J. Geophys. Res. 110, A09204 (2005). doi:10.1029/2004JA010815

Van Dierendonck, A.J., Klobuchar, J., Hua, Q.: Ionospheric scintillation monitoring using commercial single frequency C/A code receivers. In: Proc. ION GPS-93, pp. 1333-1342. The Institute of Navigation, Arlington (1993)

Wernik, A.W., Alfonsi, L., Materassi, M.: Ionospheric irregularities, scintillation and its effect on systems. Acta Geophys. Pol. 52(2), 237-249 (2004)

Wernik, A.W., Alfonsi, L., Materassi, M.: Scintillation modeling using in situ data. Radio Sci. 42 (2007). doi:10.1029/2006RS003512 